\title{
Prediction of Behavioral T/C Levels in Cochlear Implant Patients Based Upon Analysis of Electrode Impedances
}

\author{
A. Zarowski, MD ${ }^{1, *} \quad$ A. Molisz, MD ${ }^{2, *} \quad$ E. Cardinael, BaA ${ }^{1} \quad$ A. Vermeiren ${ }^{1} \quad$ T. Theunen, BaA ${ }^{1}$ \\ L. De Coninck, $\mathrm{BaA}^{1} \quad$ L. Theuwis, $\mathrm{BaA}^{1} \quad$ J. Siebert, $\mathrm{MD}^{2} \quad$ F.E. Offeciers, $\mathrm{MD}^{1}$ \\ ${ }^{1}$ European Institute for ORL-HNS, AZ Sint-Augustinus Hospital, \\ Antwerp, Belgium \\ ${ }^{2}$ Department of Family Medicine, Medical University of Gdansk, \\ Gdansk, Poland \\ J Am Acad Audiol 2020;31:674-679. \\ Address for correspondence A. Zarowski, MD, \\ Andrzej.Zarowski@GZA.be
}

\begin{abstract}
Background Fitting cochlear implants in babies and noncooperative patients is cumbersome and time consuming. Therefore, objective parameters have been sought in order to predict the subjective threshold $(T)$ and maximum comfort $(C)$ levels. Measurements of the electrically evoked compound action potentials (ECAPs) have been widely used for this purpose, yet the correlation between these objective measures and the subjective $\mathrm{T} / \mathrm{C}$ levels is weak to moderate.

Purpose This article aims (1) to evaluate correlations between the subjective parameters of the fitting maps such as thresholds ( $T$ level) and maximum comfort levels (C level), the impedance of the electrode contacts, and the ECAP thresholds, and (2) to compare the value of the electrode impedances and the ECAP measures for prediction of the $\mathrm{T} / \mathrm{C}$ levels.

Research Design Case review study in a quaternary otologic referral center.

Study Sample Ninety-eight consecutive Cl patients were enrolled. The average age of the patients was 49 years. All patients were users of the Nucleus 24RECA (Freedom, Contour Advance-of-Stylet electrode) cochlear implant.

Data Collection and Analysis Data on impedance of the electrode contacts and the behavioral $\mathrm{T} / \mathrm{C}$ levels at the first fitting session ( $2-5$ weeks after surgery) and at the 5 th fitting session (4-6 months after surgery) have been retrospectively collected in 98

Keywords

- cochlear implants

- impedance

- ECAP

- tNRT

- T/C levels

- correlation consecutive $\mathrm{Cl}$ patients. Additionally, the intraoperative impedance values and the ECAP thresholds (tNRT) have been recorded.

Results Impedances of electrode contacts show significant strong negative correlations with the stabilized $\mathrm{T} / \mathrm{C}$ levels at 4 to 6 months after implantation and are an important predictor for the behavioral T/C levels. They can explain $R^{2}=28$ to $41 \%$ of the variability of the behavioral $\mathrm{T} / \mathrm{C}$ levels. In multiple regression analysis electrode contact impedances can explain twice as much of the variability of the stabilized $\mathrm{T} / \mathrm{C}$ levels than the tNRT values. The electrode impedances together with the tNRT values
\end{abstract}

\footnotetext{
* The first two authors contributed equally to this manuscript.
}

received

December 2, 2019

accepted after revision

February 14, 2020

published online

February 8, 2021
(C) 2021. American Academy of

Audiology. All rights reserved.

Thieme Medical Publishers, Inc.,

333 Seventh Avenue, 18th Floor,

New York, NY 10001, USA
Dol https://doi.org/ 10.1055/s-0040-1718701. ISSN 1050-0545. 
are able to explain $R^{2}=37$ to $40 \%$ of the global variability of the T/C levels while the tNRT thresholds solely are able to explain only $R^{2}=5$ to $14 \%$ of the $T / C$ levels variability. Conclusion Impedances of electrode contacts correlate strongly with the stabilized behavioral $\mathrm{T} / \mathrm{C}$ levels and may be used as an objective measure for fitting of cochlear implants.

Programming of cochlear implants (CIs) in babies and noncooperative patients is cumbersome and time consuming. Therefore, objective parameters have been sought to predict the subjective threshold (T) and maximum comfort (C) levels.

Measurements of the electrically evoked compound action potentials (ECAPs) have been widely used for this purpose. Numerous researchers reported weak to moderate correlations between the ECAP thresholds and the behavioral T/C levels; however, the contribution of the ECAP thresholds for explaining the fitting maps (MAPs) remains unconvincing. ${ }^{1-4}$ The analogical role of impedances was not observed previously. 5,6

This study has been undertaken to evaluate the possible correlation between the subjective parameters of the fitting maps such as $\mathrm{T} / \mathrm{C}$ levels and the impedance of the electrode contacts. The measurements were performed in a large group of patients implanted with Nucleus Freedom CIs allowing for very accurate impedance measurements.

The study objectives were: (1) to evaluate correlations between the subjective parameters of the fitting maps such as thresholds (T level) and maximum comfort levels ( $\mathrm{C}$ level), the impedance of the electrode contacts, and the ECAP thresholds, and (2) to compare the value of the electrode impedances and the ECAP measures for prediction of the T/C levels.

\section{Material and Methods}

Data on impedance of the electrode contacts and the behavioral $\mathrm{T} / \mathrm{C}$ levels at the first fitting session (2-5 weeks after surgery) and at the fifth fitting session (4-6 months after surgery) have been collected in 98 consecutive CI patients. Additionally, the intraoperative impedance values and the ECAP thresholds ( $t N R T$ ) have been recorded.

The average age of the patients was 49 years. - Fig. 1 presents the histogram of age distribution in the study group. The binominal age distribution was reflected neither in the distribution of the electrode impedances nor in the values of the $\mathrm{T} / \mathrm{C}$ levels and the tNRT thresholds, so that the analysis of correlations could be correctly performed.

To be included in the study all patients had to be users of the Nucleus 24RECA (Freedom, Contour Advance-of-Stylet electrode) CI. This was necessary for the consistency of the comparisons of the $\mathrm{T} / \mathrm{C}$ levels. Included patients needed to have all functioning electrode contacts with impedances in the normal range and be regular users of the implant at least until the fifth fitting session 4 to 6 months after surgery.

The exclusion criteria covered no or irregular use of speech processor, inactivation of some electrode contacts, or irregular electrode conditions.

All implantations were performed via the posterior tympanotomy/cochleostomy approach with Contour Advance-

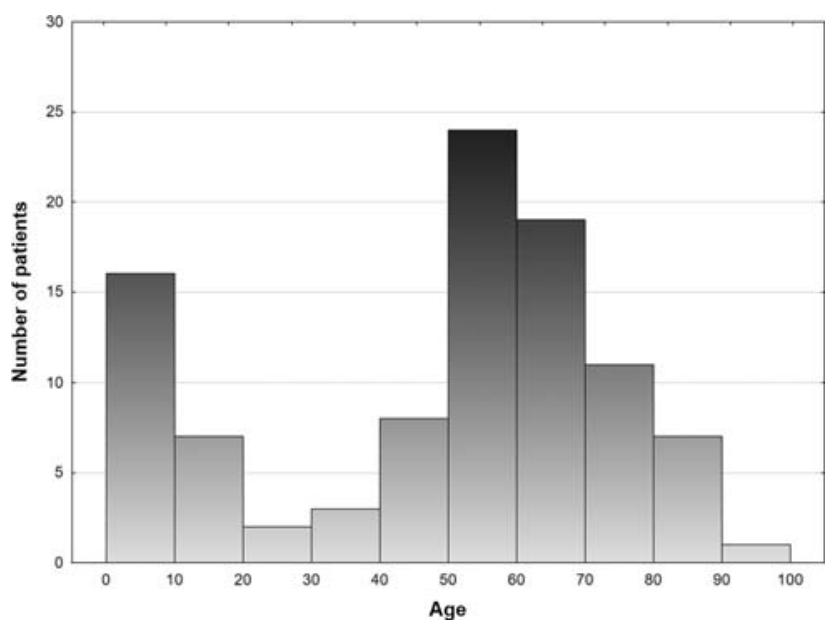

Fig. 1 Histogram of age distribution in the study group.

of-Stylet electrode insertion technique. Topical steroids (triamcinolone acetonide, Volon A) mixed with hyaluronic acid (Healon) have been used to cover the cochleostomy site and to soak the electrode before insertion into the cochlea.

The programming of the processor was begun after full healing of the retroauricular wound between 2 and 5 weeks postoperatively for the first implant programming session. In small children during the first implant programming session, the $\mathrm{T} / \mathrm{C}$ level profiles were programmed based on the results of the intraoperative tNRT measurements. The $\mathrm{T}$ levels were basically programmed $30 \mathrm{CL}$ (Cochlear current levels) under the tNRT thresholds and the C levels $15 \mathrm{CL}$ above the T level, with progressive maps created every $5 \mathrm{CL}$. Visual reinforcement audiometry and behavioral observation were utilized for all consecutive implant programming sessions to adapt the $\mathrm{T} / \mathrm{C}$ levels created during the first session. In adult patients the $\mathrm{T} / \mathrm{C}$ levels were adjusted according to the behavioral responses using the loudness growth charts with the tNRT values used as only an approximate reference for the profile of the $\mathrm{T} / \mathrm{C}$ levels at the first fitting session. At the end of programming all $\mathrm{T} / \mathrm{C}$ levels the Clevels have been globally lowered by $15 \mathrm{CL}$ and then globally raised in function of the perceived loudness.

In all pediatric and adult patients all channels were measured separately and no streamlined fitting protocols were used. In the course of subsequent implant programming sessions, the $\mathrm{T} / \mathrm{C}$ levels became progressively adjusted to the behavioral responses both for the pediatric and for the adult populations.

Impedance measurements were performed using the proprietary software of the Cochlear company registering the voltage at the end of the first phase of the 25 - $\mu$ s per phase current pulses with an amplitude of $100 \mathrm{CL}$. Monopolar $1+2$ and common ground (CG) electrode configurations were 
analyzed. The intraoperative ECAP thresholds (tNRT) were evaluated using the Auto-NRT program of Cochlear using the forward-masking paradigm of the Custom Sound EP and the current pulses of 25 - $\mu$ s per phase with the repetition rate of 250 pulses per second. The behavioral evaluation of the $\mathrm{T}$ levels and the $C$ levels was done with the Custom Sound version 3.0 fitting program, using the 25 - $\mu$ s current pulses with the repetition rate of 900 pulses per second per channel. All implant fittings have been performed by only two experienced $\mathrm{CI}$ audiologist using exactly the same fitting protocol.

Analyses were performed separately for the basal, midportion, and apical electrodes. As representative for the basal electrodes an average result for electrode contacts 3, 4, and 5, for the mid-portion electrodes an average result for electrode contacts 11,12 , and 13 , and for the apical electrodes an average result for electrode contacts 19,20 , and 21 was calculated.

Statistical analysis of the correlations between the electrode impedances and the $\mathrm{T} / \mathrm{C}$ levels obtained from first and fifth fitting sessions was performed. Additionally, the relationship between the intraoperatively measured electrode impedances and the intraoperative tNRTs was assessed. Pearson's product-moment correlation coefficient was calculated for all the correlations. Multiple regression analysis was used to compare the value of the electrode impedances and the ECAP measures for prediction of the T/C levels.

All data were collected at the European Institute for ORLHNS in Antwerp, Belgium. Appropriate approval of the institutional Ethical Commission has been obtained. Statistica 10 by StatSoft was used for the statistical analysis.

\section{Results}

Correlations between the Electrode Impedances and the Behavioral T/C Levels

At the first fitting session, only weak negative correlations between the electrode impedances and the behavioral $\mathrm{T}$ and
C levels could be found for all electrode contacts (Pearson's correlation coefficient for the T levels was between -0.28 and -0.31 and for the $C$ levels between -0.26 and -0.30 ).

However, at the fifth fitting session strong negative correlations have been found between these parameters (Pearson's correlation coefficient values range from $r=-$ 0.54 to -0.68 ). All of these correlations were highly statistically significant with $p<0.001$ and comparable for the basal, the mid-portion electrodes, and the apical electrodes.

The results for the fifth fitting session are graphically presented in - Fig. 2 . The Pearson's correlation coefficient $R$ values (together with the $R^{2}$ ) are shown in - Table 1. Measurement of the impedances in the CG configuration gave comparable results of correlation with the $\mathrm{T} / \mathrm{C}$ levels (not shown in the figures).

\section{Correlations between the Intraoperative Electrode Impedances and the ECAP Thresholds (tNRT)}

For all electrode contacts, weak to moderate positive correlations between the intraoperatively measured electrode impedances and the intraoperative ECAP thresholds (tNRT) have been found (-Fig. 3). For the basal electrodes, the value of Pearson's correlation coefficient $R=0.26$, for the mid-portion electrodes $R=0.30$, and for the apical electrodes $R=0.36$. All these correlations were significant with $p<0.05$.

\section{Correlations between the ECAP Thresholds (tNRT) and the T/C Levels}

The relation between the ECAP thresholds (tNRT) and the T/C levels is complex. At the first fitting sessions moderate correlations can be observed for the apical and the basal electrodes. This is due to the fact that at initial fittings the $\mathrm{T} / \mathrm{C}$ levels are defined based on the profile obtained with the tNRT measurement. During the fitting process, the $\mathrm{T}$ and $\mathrm{C}$ levels are adjusted to the behavioral responses of the patients and rely less on the tNRT profiles. This leads to weakening of
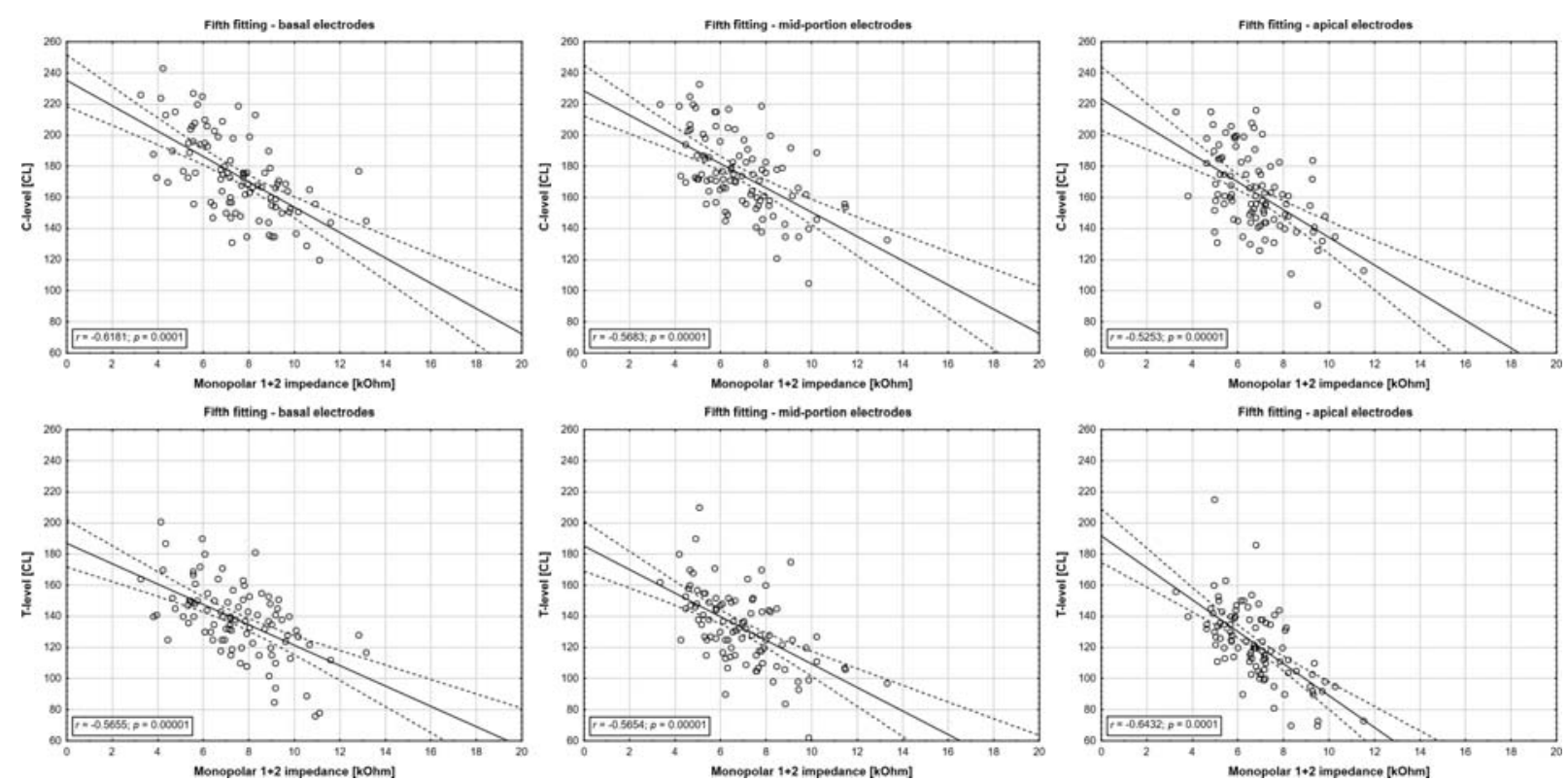

Fig. 2 Correlation between the electrode impedances (monopolar $1+2$ configuration) and the behavioral $\mathrm{T}$ and $\mathrm{C}$ levels for the basal, midportion, and the apical electrodes measured at the fifth fitting session. 
Table 1 Correlation between the electrode impedances and the behavioral T and C levels (values of Pearson's correlation $R$ coefficient at the fifth fitting session)

\begin{tabular}{|l|l|l|l|}
\hline Electrode contact & Configuration & Behavioral T level $[R]$ & Behavioral C level $[R]$ \\
\hline Basal & Monopolar $1+2$ & $-0.57^{\mathrm{a}}\left(R^{2}=0.32\right)$ & $-0.62^{\mathrm{a}}\left(R^{2}=0.38\right)$ \\
\hline Mid-portion & Monopolar $1+2$ & $-0.57^{\mathrm{a}}\left(R^{2}=0.32\right)$ & $-0.57^{\mathrm{a}}\left(R^{2}=0.32\right)$ \\
\hline Apical & Monopolar $1+2$ & $-0.64^{\mathrm{a}}\left(R^{2}=0.41\right)$ & $-0.53^{\mathrm{a}}\left(R^{2}=0.28\right)$ \\
\hline
\end{tabular}

${ }^{\mathrm{a}} R$ coefficients with statistical significance $p<0.05$ (actually the correlations were all highly significant with $p<0.0001$ ).
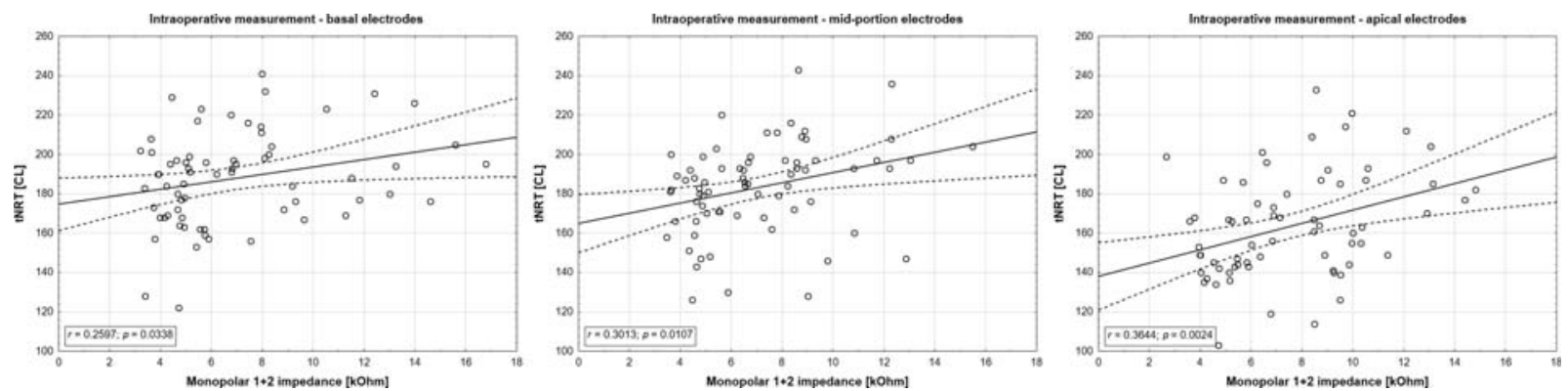

Fig. 3 Correlation between the intraoperatively measured electrode impedances (monopolar $1+2$ configuration) and the intraoperative tNRT thresholds for the basal, mid-portion, and apical electrodes.

the correlations between the tNRT and the T/C levels measured at the fifth fitting session.

No correlations at all have been found for the mid-portion electrodes, neither for the first nor for the fifth fitting session.

We can also note large variability of the $\mathrm{T} / \mathrm{C}$ levels for each measured tNRT value. This together with the weak correlations seriously limits the clinical usefulness of tNRTs for programming of the fitting maps.

The scattergrams and the results of the correlations analysis between the ECAP thresholds (tNRT) and the T/C levels are graphically presented in -Fig. 4.
The Pearson's correlation coefficient $R$ (together with the $R^{2}$ ) values for the correlation between the tNRT and the T/C levels are shown in -Table 2.

\section{Multiple Regression Analysis}

To compare the relative contributions of the tNRT levels and the electrode impedances for explaining the variability of the measured $\mathrm{T} / \mathrm{C}$ levels, the multiple regression analysis was performed. In this analysis, the variability $\mathrm{T}$ or the $\mathrm{C}$ levels was explained by variability of only two variables, the tNRT threshold and the electrode impedance,
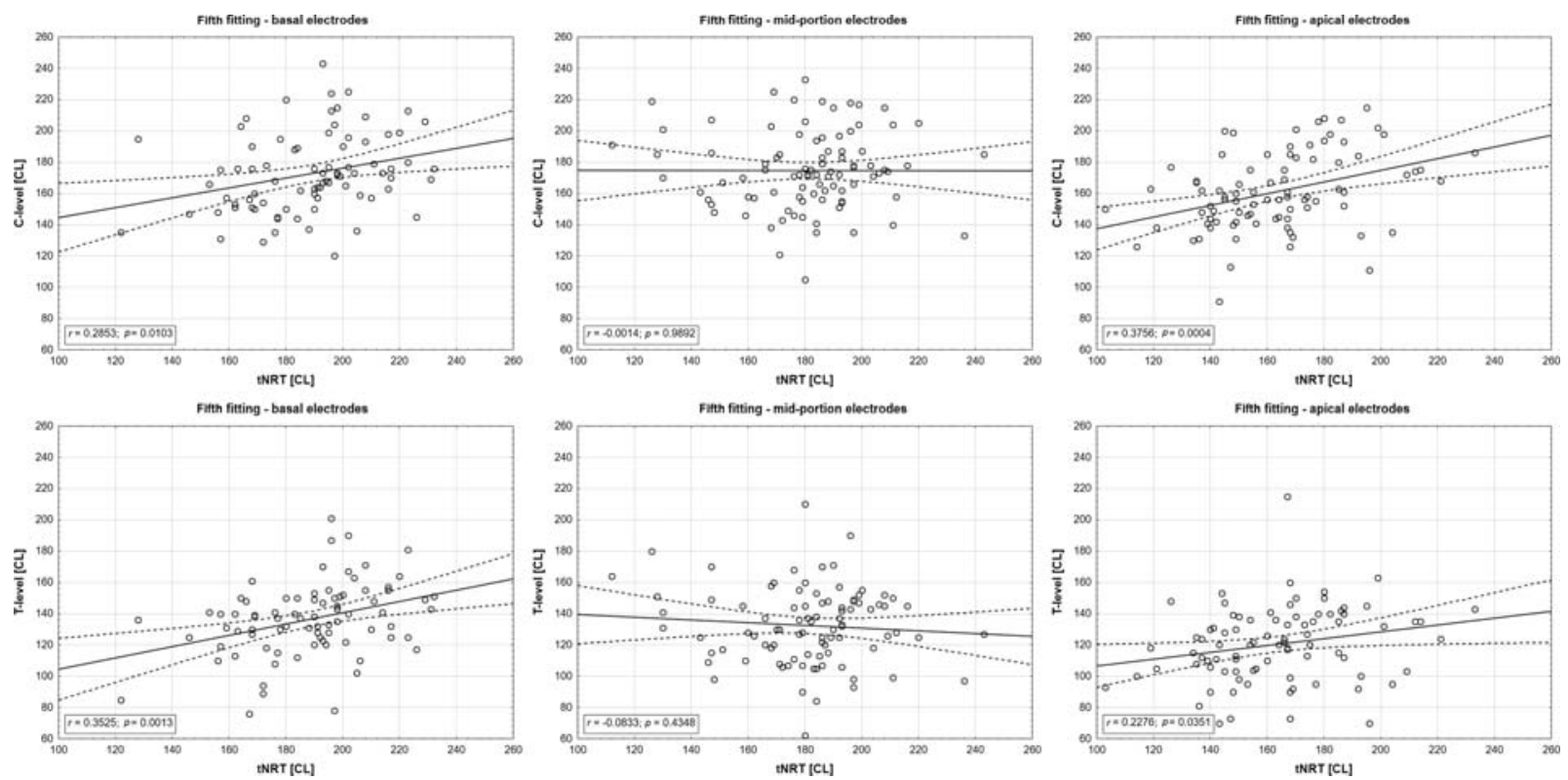

Fig. 4 Correlation between the intaoperatively measured tNRT thresholds and the behavioral T and C levels for the basal, mid-portion, and apical electrodes measured at the fifth fitting session. 
Table 2 Correlation between the measured tNRT values and the behavioral T and C levels (values of Pearson's correlation $R$ coefficient at the fifth fitting session)

\begin{tabular}{|l|l|l|}
\hline tNRT & $\begin{array}{l}\text { Behavioral } \\
\text { T level }[R]\end{array}$ & $\begin{array}{l}\text { Behavioral } \\
\text { C level }[R]\end{array}$ \\
\hline Basal electrodes & $0.35^{\mathrm{a}}\left(R^{2}=0.12\right)$ & $0.29^{\mathrm{a}}\left(R^{2}=0.08\right)$ \\
\hline $\begin{array}{l}\text { Mid-portion } \\
\text { electrodes }\end{array}$ & $-0.08\left(R^{2}=0.01\right)$ & $-0.001\left(R^{2}=0\right)$ \\
\hline Apical & $0.23^{\mathrm{a}}\left(R^{2}=0.05\right)$ & $0.38^{\mathrm{a}}\left(R^{2}=0.14\right)$ \\
\hline
\end{tabular}

${ }^{\mathrm{a}} R$ coefficients with statistical significance $p<0.05$.

Table 3 Beta and $R^{2}$ coefficients resulting from the multiple regression analysis

\begin{tabular}{|l|l|l|l|}
\hline 三eta weight & tNRT & Impedance & $R^{2}$ \\
\hline T level 1st fitting & 0.39 & -0.26 & 0.23 \\
\hline C level 1st fitting & 0.36 & -0.28 & 0.21 \\
\hline T level 5th fitting & 0.25 & -0.56 & 0.37 \\
\hline C level 5th fitting & 0.30 & -0.57 & 0.40 \\
\hline
\end{tabular}

according to the multiple regression equation: $T / C$ level $=$ $b 1^{*} t N R T+b 2 *$ Impedance $+A$

The Beta and $R^{2}$ coefficients resulting from the multiple regression analysis have been presented in - Table 3 . At the first fitting session, when the $\mathrm{T} / \mathrm{C}$ levels are defined based on the profile obtained with the tNRT measurement the influence of the tNRT obviously overweighs the role of the impedance of the electrode contacts. This artificial relation, leads to relatively low values of the $R^{2}$ coefficient, meaning that at the first fitting session combination of the influences of the tNRT with electrode impedances can explain only 21 to $23 \%$ of the variability of the $\mathrm{T} / \mathrm{C}$ levels.

However, at the fifth fitting session the influence of the electrode impedance strongly (by the factor 2) overweighs the relative contribution of the tNRT values. At the fifth fitting session combination of the influences of the tNRT with electrode impedances can explain already 37 to $40 \%$ of the variability of the $\mathrm{T} / \mathrm{C}$ levels.

\section{Discussion}

The impedance of the $\mathrm{CI}$ electrodes shows significant changes during the first 4 to 12 weeks after implantation. ${ }^{7}$ These changes are caused by the surgical trauma of electrode insertion into the cochlea triggering the inflammatory reactions and subsequent healing. The inflammatory proteins ${ }^{8}$ can adhere to the electrode contacts changing their active surface and increasing the electrical impedance. Subsiding inflammation and/or start of electrical stimulation "cleans" the electrode contacts and stabilizes the impedances.

Another factor influencing the electrical impedance between the intracochlear and the reference electrodes is the current leakage through the site of cochleostomy. The healing process resulting in progressive sealing of the cochleostomy by fibrous tissue within the first weeks after implantation makes this low impedance current leakage path less pronounced and stabilizes the impedance.

Also, generalized inflammatory reactions in the body result in appearance of specific proteins in the perilymph ${ }^{9}$ that can influence the active surface and increase the impedance of the electrode contacts. This research was actually initiated by a clinical observation of increased electrical impedances measured in a few $\mathrm{CI}$ patients with signs of upper respiratory infections (URIs) with or without increased body temperature. Before URI these patients showed stable CI parameters and good results of speech discrimination with the implant, while during the URI they suddenly started to complain about excessive loudness sensations. Implant integrity checkup showed normal functioning of the devices, the only irregularity was increased impedance of the electrode contacts. Increased contact impedance has been a constant finding in all our patients with URI and suddenly increased subjective loudness sensations.

According to our knowledge, this is the first study demonstrating the importance of electrode impedances as a strong predictor for the behavioral $\mathrm{T} / \mathrm{C}$ levels. This correlation can aid in automatic fitting of CIs based on objectively measured electrophysiological parameters. Existence of this correlation has never been demonstrated before. Greisiger et al reported relation between impedances and behavioral parameters only in some patients; however, no significant correlations were found in the whole group. ${ }^{5}$ King et al presented no significant predictive value of electrode impedances for objective setting of behavioral comfort levels. ${ }^{6}$

Our study shows that the electrode impedance itself can explain 28 to $41 \%$ of the variability of the stabilized T/C levels at the fifth fitting session 4 to 6 months postoperatively. This is a very significant influence taking into consideration that cognitive parameters, previous experience with amplification, the age of the patient, etc. also play an important role in the definition of the behavioral $\mathrm{T} / \mathrm{C}$ levels. At the fifth fitting session, the contribution of the electrode impedance is twice as important as the tNRT thresholds (in multiple regression analysis the Beta weight for the electrode impedance is twice as big as the tNRT).

For the apical and basal electrodes, the tNRT thresholds are able to explain only 5 to $14 \%$ of the T/C levels variability. Additionally, the tNRT thresholds have no value for prediction of the behavioral $\mathrm{T} / \mathrm{C}$ levels for the mid-portion electrodes where these variables are not correlated with each other. Our observations of limited role of tNRT levels in the prediction of behavioral $\mathrm{T} / \mathrm{C}$ levels are congruent with data presented by numerous authors, ${ }^{1,2}$ although this approach is still recommend for fitting of the children and noncooperative patients. $6,10,11$

Weak correlations between the tNRT levels and the behavioral $\mathrm{T} / \mathrm{C}$ levels stimulated research for additional factors that could improve the prediction power of the tNRT. For example, McKay and Smale presented the relation between the rate of stimulation and the behavioral thresholds, additionally influenced by such ECAP parameters as the ECAP amplitude growth slope and the effect of changes in interphase gap and the phase duration. ${ }^{12}$

In our study adding the information on the electrode impedances and the tNRT values in a multiple regression 
analysis additionally improves the prediction of the $\mathrm{T} / \mathrm{C}$ levels. For all electrodes analyzed together 37 to $40 \%$ of the global variability of the $\mathrm{T} / \mathrm{C}$ levels can be explained.

The factors that allowed demonstration of the correlations between the electrode impedances and the $\mathrm{T} / \mathrm{C}$-levels are following: (1) relatively large group of patients implanted with the same type of $\mathrm{Cl}$ system; (2) uniform and meticulous fitting procedure performed separately for all channels by only two experienced audiologists using the same fitting protocol; (3) availability of the impedance values for each fitting session; and (4) calculation of correlations for stabilized T/C-levels at the $5^{\text {th }}$ fitting session 4-6 months after implantation ${ }^{7}$ when the $\mathrm{T} / \mathrm{C}$ levels are based purely on the behavioral reactions and not anymore on tNRT.

\section{Conclusion}

1. Impedances of electrode contacts show significant strong correlations with the stabilized T/C levels at 4 to 6 months after implantation and are an important predictor for the behavioral T/C levels. They can explain $R^{2}=28$ to $41 \%$ of the variability of the behavioral $\mathrm{T} / \mathrm{C}$ levels and may be used as an objective measure for fitting of CIs.

2. In multiple regression analysis, electrode contact impedances can explain twice as much of the variability of the stabilized T/C levels than the tNRT values.

3. The electrode impedances together with the tNRT values are able to explain $R^{2}=37$ to $40 \%$ of the global variability of the $\mathrm{T} / \mathrm{C}$ levels while the tNRT thresholds solely are able to explain only $R^{2}=5$ to $14 \%$ of the T/C levels variability.

\section{Conflict of Interest}

Drs. Offeciers and Zarowski have a patent pending No. WO2019229162A1 (Method for deriving information for fitting a cochlear implant). The remaining authors have no conflict of interest.

\section{Acknowledgments}

The study protocol was designed and performed as result of collaboration between the two mentioned centers.

\section{References}

1 Holstad BA, Sonneveldt VG, Fears BT, et al. Relation of electrically evoked compound action potential thresholds to behavioral Tand C-levels in children with cochlear implants. Ear Hear 2009;30 (01):115-127

2 Potts LG, Skinner MW, Gotter BD, Strube MJ, Brenner CA. Relation between neural response telemetry thresholds, T- and C-levels, and loudness judgments in 12 adult nucleus 24 cochlear implant recipients. Ear Hear 2007;28(04):495-511

3 Tao Y, Zheng Y, Wang K, Meng Z, Xu K, Xie T. Compare NRT threshold and behavior T-level in cochlear implant patients [in Chinese]. Lin Chung Er Bi Yan Hou Tou Jing Wai Ke Za Zhi 2011;25 (04):162-165

4 Vlahović S, Šindija B, Aras I, Glunčić M, Trotić R. Differences between electrically evoked compound action potential (ECAP) and behavioral measures in children with cochlear implants operated in the school age vs. operated in the first years of life. Int J Pediatr Otorhinolaryngol 2012;76(05):731-739

5 Greisiger R, Shallop JK, Hol PK, Elle OJ, Jablonski GE. Cochlear implantees: analysis of behavioral and objective measures for a clinical population of various age groups. Cochlear Implants Int 2015;16(Suppl 4):1-19

6 King JE, Polak M, Hodges AV, Payne S, Telischi FF. Use of neural response telemetry measures to objectively set the comfort levels in the Nucleus 24 cochlear implant. J Am Acad Audiol 2006;17 (06):413-431, quiz 462

7 Molisz A, Zarowski A, Vermeiren A, et al. Postimplantation changes of electrophysiological parameters in patients with cochlear implants. Audiol Neurotol 2015;20(04):222-228

8 Schmitt H, Roemer A, Zeilinger C, et al. Heat shock proteins in human perilymph: implications for cochlear implantation. Otol Neurotol 2018;39(01):37-44

9 Lin HC, Ren Y, Lysaght AC, Kao SY, Stankovic KM. Proteome of normal human perilymph and perilymph from people with disabling vertigo. PLoS One 2019;14(06):e0218292

10 Mittal R, Panwar SS. Correlation between intra-operative high rate neural response telemetry measurements and behaviourally obtained threshold and comfort levels in patients using Nucleus 24 cochlear implants. Cochlear Implants Int 2009;10(02):103111

11 Muhaimeed HA, Anazy FA, Hamed O, Shubair E. Correlation between NRT measurement level and behavioral levels in pediatrics cochlear implant patients. Int J Pediatr Otorhinolaryngol 2010;74(04):356-360

12 McKay CM, Smale N. The relation between ECAP measurements and the effect of rate on behavioral thresholds in cochlear implant users. Hear Res 2017;346:62-70 